\title{
Retained second molars: therapeutic approach
}

\author{
J. Vexiau', B. Castaneda ${ }^{2,3,4}$ \\ 1 Dental surgeon, CECSMO 4 Paris VII, Pitié-Salpêtrière Hospital Group \\ 2 Dentofacial Orthopedics Dept., Paris, France \\ 3 Laboratory of Molecular Oral Pathophysiology, INSERM UMR 1138, Paris, France \\ 4 Faculty of Odontology, University of Antioquia, Colombia
}

\begin{abstract}
Second molar eruption abnormalities are quite rare, and thus there is no precise consensus protocol for their setting. Several therapeutic solutions will be presented here: purely orthodontic, or associating orthodontics and surgery.
\end{abstract}

\section{KEYWORDS}

Permanent second molar, primary failure of eruption, mechanical failure of eruption, treatment of retained molars

\section{INTRODUCTION}

Second molar eruption abnormality is fairly rare, but seems to be slightly increasing ( $1 \%$ according to Cho, 20083). Several etiologies have been suggested. Locally, a trend away from premolar extraction and in favor of space-maintaining treatments could partly explain the increase ${ }^{14}$.

Various treatments are available for second molar eruption pathology. Etiology plays a role in determining treatment.

Primary failure of eruption (PFE), defined by Proffit in 1981'12, is to be distinguished from mechanical failure of eruption (MFE), defined by Suri in $2002^{16}$. Diagnosis, however, is often difficult, and it is often attempted to position the retained tooth.
In case of MFE, or when a diagnosis of PFE is uncertain, several solutions may be considered: orthodontic or surgical treatment, a combination of the two, or abstention. Conversely, if the diagnosis of PFE is certain, surgery is preferable, associated to orthodontic treatment or not.

Other factors are to be taken into account in guiding treatment (see below).

As these abnormalities are not widespread, there is no well-defined therapeutic protocol. Various solutions have been described: orthodontic, or combining orthodontics and surgery, as with miniscrews or miniplates. Although based on simple case reports or still in the experimental stages, they will be presented here, to help clinicians in their practice. 


\section{FACTORS INFLUENCING THERAPEUTIC DECISION-MAKING}

Retention is diagnosed on several criteria, including progression of the contralateral tooth or occlusal level of the second as compared to the first molar if the tooth has emerged into the oral cavity without meeting the first molar occlusion plane.

Treatment is guided by several factors, depending on patient age, root edification stage (open or closed

\section{ORTHODONTIC SOLUTIONS}

Many authors have described a variety of means of positioning retained or impacted second molars in the arcade. Surgical exposure is usually required, to allow a button, tube or hook to be attached for traction. In most cases, and especially in the mandibular arcade, the adjacent third molar, when present, is extracted.

There have been many more reports of retained or iimpacted mandibular than maxillary second molars.

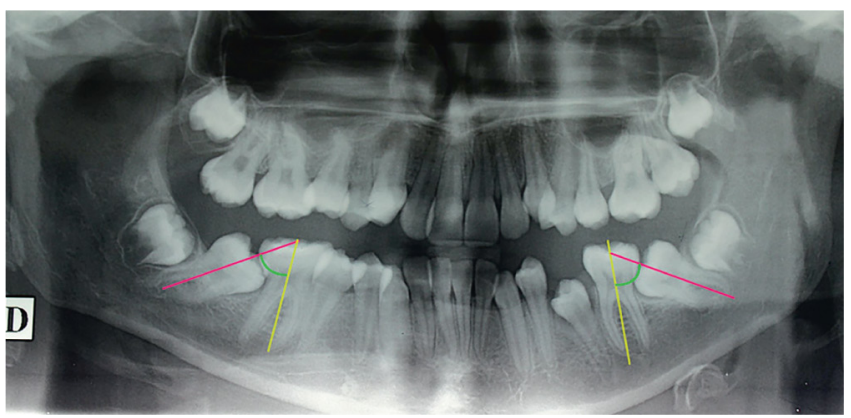

Figure 1

Panoramic view: Angélique, 15 years old. Class I/ division 1 associated with retention of mesially inclined mandibular second molars at an angle $>25^{\circ}$ to the first molar axis (Thanks to Dr Sylvie Legris). dental apex), tooth inclination, presence of adjacent third molar, associated first molar retention, intra-oral visibility, etc.

The type of bone tissue around the tooth also plays a part in choice of treatment: teeth retained or impacted in the mandible are surrounded by much denser bone tissue than in the maxilla.

Moreover, most reports concerned second molars in mesial inclination, although this is far from being the most frequent state of affairs (Fig. 1).

Before detailing the various systems for setting retained or impacted second molars, some biomechanical reminders will be given.

When distalizing force is applied to a mesially inclined molar, this induces a momentum leading to distal version of the tooth. Figure 2 shows that

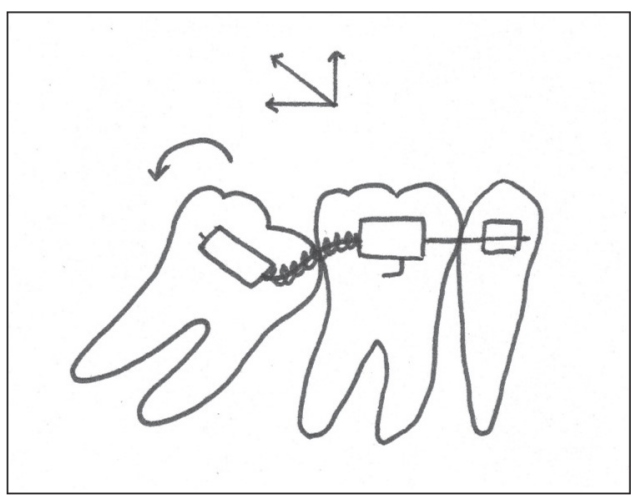

Figure 2

Distalizing and extrusive force applied by the orthodontic system, with anti-clockwise rotation. 
the force applied by the orthodontic system sets up an anti-clockwise moment that uprights the mesially inclined tooth; this also induces egression, which may need controlling.

Lau et al. ${ }^{6}$ described what they called "routine" straight arch techniques for setting mesially inclined retained mandibular second molars: after surgically releasing the distal side of the tooth, they insert an compressed open spring between the first and second molars, with the tube glued to the latter at $90^{\circ}$ to the occlusal plane so as to promote uprighting. If the tube were glued parallel to the occlusion plane, the tooth would basically move backward, and the distalization force would induce a

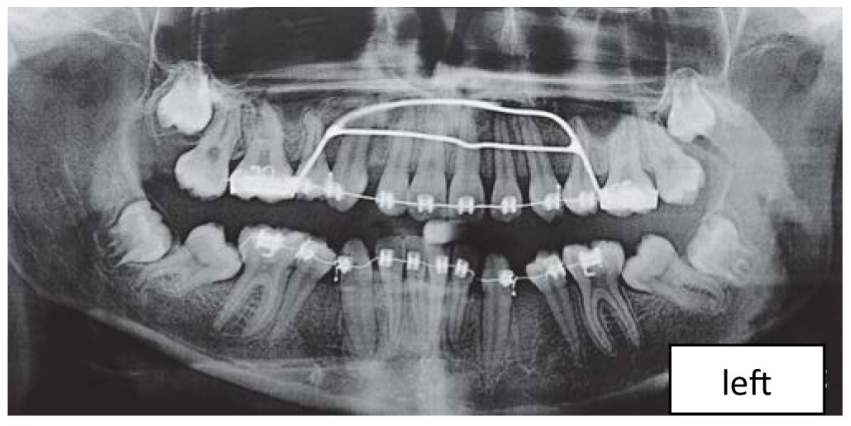

a

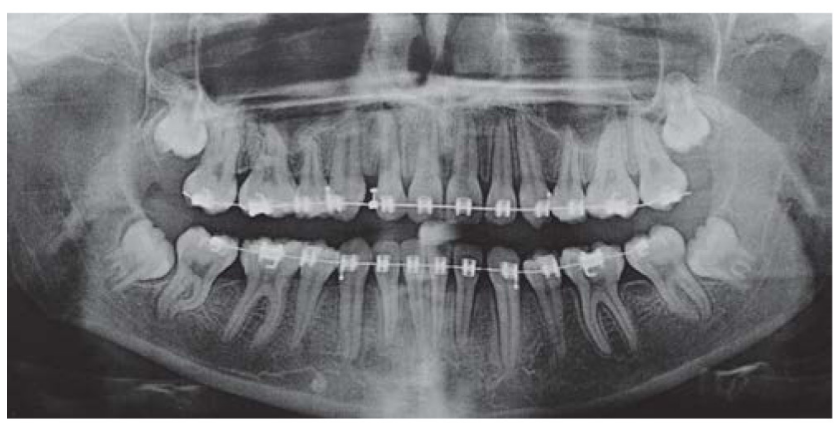

b

Figure 3

a) 37 and 47 retained despite extraction of 34 and 44 . b) 8 months later, 37 and 47 well-positioned in the arcade. (Thanks to Dr Sylvie Legris). moment tending to upright the mesially inclined tooth; fixing the tube at $90^{\circ}$ creates a much stronger moment and greater uprighting displacement. During treatment, the straight arch does not include the first molar, so as to increase the flexibility of the arch and reduce force so as to avoid unwanted effects such as root resorption or problems at the attachment.

The authors add that the posterior maxillary occlusion planes had to be raised to enable molar uprighting without occlusal interference. In this case, this did not impair the results of treatment.

Elastic bands (e.g., criss-cross) may be used as back-up to complete occlusion of the repositioned second molar.

Elevation planes on the posterior teeth may, in some patients, especially those with facial hyperdivergence, induce unwanted mandibular opening and/or gap.

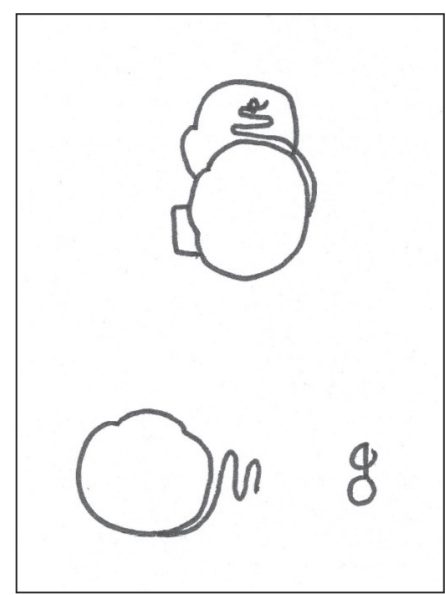

Figure 4

Below, occlusal view of ring with welded spring and custom-made hook; above, occlusal view of system in position, with ring fixed to the molar mesial to the tooth to be uprighted, hook glued to tooth to be uprighted and spring in position. 
Ziegler $^{18}$ likewise reported a system using a compressed open spring between the first mandibular molars and the second, mesially inclined in retention, onto which an attachment system was fitted. He called this "versatile molar uprighting mechanics" and developed it over a period of some 15 years, ending up with a system using self-ligating brackets. A sectional bracket is placed between the first molar and the second molar, to be uprighted, (or between the second and third, as Ziegler also applied this system for third molars), and a compressed screw uprights the mesially inclined molar. In one of his cases, like Lau et al., he did not include the first molar to help upright the second. Anchorage involved the whole

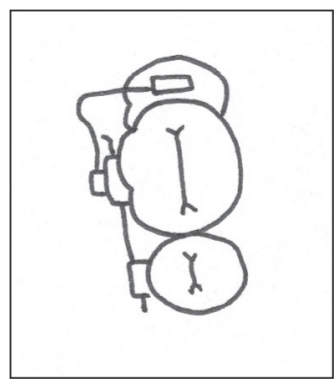

Figure 5

Modified Loca system.

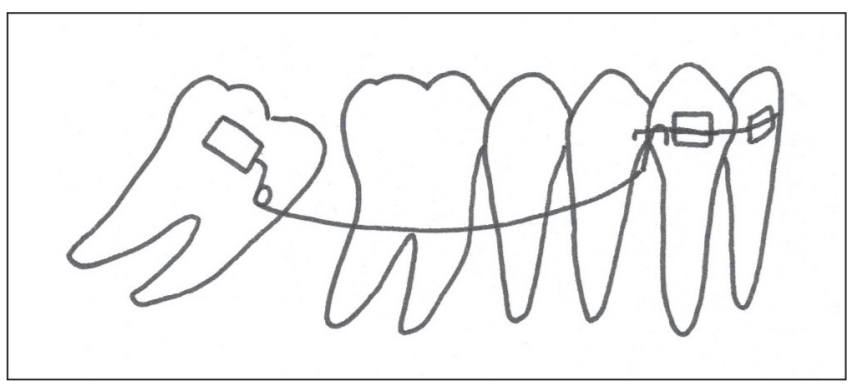

Figure 6

Sawicka technique. arcade, with a $.016 \times .022$ or $.019 \times$ .025 steel bracket, depending on the type of attachment. The reported cases did not involve third molar germectomy to upright the second molar.

Another example concerns a young patient presenting with dentomaxillary dysharmony and retained second molars, managed by extraction of the first maxillary and second mandibular premolars. Resolution of posterior crowding allowed spontaneous progression of both second molars. In other cases of dentomaxillary dysharmony, premolar extraction is not enough to enable spontaneous progression. Such, for example, was the case of the patient shown in Figure 1, with retained second molars. The first premolars were extracted. The second mandibular molars were in retention, but with the distal part of the vestibular side accessible, and could be managed by glued minitubes and a .014 copper NiTi arch. In a second step, a .018 steel arch with coiled loops was fitted between the first and second molars. This type of system has the same effect as a spring, but with better mechanical control. The entire arcade is included, ensuring anchorage while the second molars are uprighted (Fig. 3a and b).

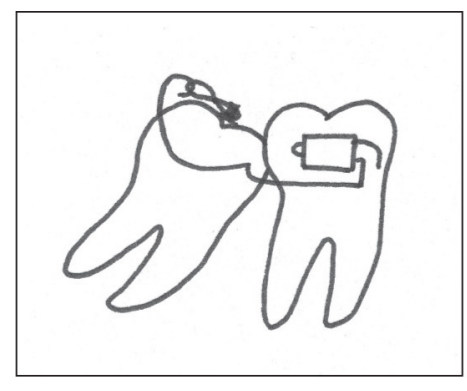

a

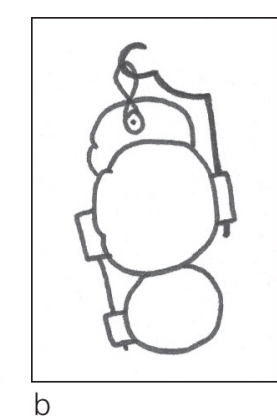

Figure 7 a) Santoro et al., lingual view: b) occlusal view. 
Miao et $a .^{8}$ and Ma et al. ${ }^{7}$ described a technique applied essentially for mandibular third molars but also sometimes successfully for mandibular second molars (Fig. 4).

Miao et al. fitted a custom-made hook to the molar to be repositioned, with a ring on the adjacent tooth and a .018 steel spring welded to the ring; the spring exerted a distalizing but also slightly extrusive force on the tooth.

Ma et al. fitted a custom-made hook to the tooth to be uprighted after surgical exposure; a ring with a .016 steel spring was fixed to the adjacent molar, intended to exert a purely distalizing force. In a second step, the tooth was extruded using a $.017 \times .025$ TMA sectional bracket, with $.019 \times .025$ steel anchorage on the 4 teeth anterior to the tooth to be uprighted. Finally, the position of the tooth was adjusted. The technique was reported for third molars requiring uprighting ahead of extraction, but was also successfully used on some mandibular second molars.

Loca's system, modified (Celli et al. ${ }^{2}$ ), and another system described by Paik et al. ${ }^{10}$ use an NiTi arch with extra wire; as it tries to recover its initial shape, the arch uprights the molar (Fig. 5). This again concerns retained mandibular second molars with mesial inclination.

In the modified Loca system, an NiTi sectional bracket is positioned between the first and second molars and the whole arcade, except the second molars to be uprighted, is included by a distally curved .020 Australian wire, and a glued lingual contention reinforces the anchorage and focuses force on the molar to be uprighted. The authors specify that $.019 \times .025$ steel would be better than the .020 Australian wire, to optimize anchorage and minimize incisor vestibular drift.

Sawicka et al. ${ }^{14}$ described a system initially including just the mandibular incisor-canine sector and 2 TMA sectional brackets fixed distally to the canines and linked to the 2 mesially inclined molars, creating a strong lever arm (Fig. 6). The 6 anterior teeth support a $.017 \times .025$ steel sectional bracket in .018 grooves; a figure-ofeight ligature connects them, and anchorage is completed by a glued contention. Once the molars have begun to straighten, the entire arcade is included.

Bach ${ }^{1}$ described a technique in which a .013 copper NiTi arch is inserted distally, with a loop to the tube on the adjacent tooth. This distal insertion is intended to exert both distalizing and intruding force on the tooth, whereas many other systems that have been reported exert an extruding force (which accounts for the need for elevation planes on the posterior teeth to enable distal rotation of the second molar, but with a risk of creating a gap, as mentioned above).

Santoro et al. ${ }^{13}$ described a more original system (Fig. 7a and b) using a commercial transpalatal bar modified to fit in a palatine/lingual sheath and to reach the distal side of the retained second molar. A chain connecting the button to the hook on the modified transpalatal bar enables distalizing traction. Anchorage is provided by including the entire arcade with a steel arch. The system is thus said to be applicable to both maxilla and mandible, although the reported cases concerned mandibular molars. 
As there are few examples concerning maxillary molars in the literature, some unpublished cases of treatment of retained maxillary second molars will be described.

A patient presenting with a retained maxillary second molar underwent surgical exposure of the occlusal side of the tooth, to which a tube was glued. A $.017 \times .022$ sectional bracket with blue elgiloy loops was positioned to achieve occlusal traction, exerting a mainly extrusive force (Fig. 8a and b). As soon as clinically possible, a tube was glued onto the vestibular side, with a sectional bracket to the first molar including the tooth under traction (Fig. 8c). The wisdom tooth germ was not removed at first, in case there was ankylosis; once traction had proved successful, germectomy was performed.

Setting a maxillary second molar may in some cases require slight distal enamel reduction on the adjacent first molar, associated to orthodontic treatment. Such was the case for a young patient presenting with a right maxillary second molar in occlusion and the left retained under the distal convexity of the first molar (Fig. 9a). Mucosal release was performed, with reduction of first molar distal enamel. A minitube was fixed onto 27, for intermaxillary traction.

As the effect was insufficient, a sectional bracket was positioned from the second premolar to the second molar, with a compressed spring to distalize the tooth, associated to gentle dislocation (Fig. 9b). The anchorage was reinforced by a transpalatal Nance appliance linking the right maxillary first molar to left second premolar. A triangular vertical elastic band was associated, so that egression was limited to the second molar, without ingression of the first molar (Fig. 9c). Egression was progressive, without side-effects (Fig. 9d and e).

\section{SOLUTIONS COMBINING ORTHODONTICS AND SURGERY}

Surgery may be considered: gentle dislocation (notably in PFE, where the risk of ankylosis is high), or extraction if tooth positioning seems too complicated or impossible.

In other cases, treatment may associate orthodontics to miniscrews or miniplates, or to dislocation.

\section{Miniscrews and orthodontics}

Many authors have reported use of miniscrews in impacted or retained second molars. Traction is usually applied directly by the miniscrew, which is in other cases used to reinforce anchorage.

In impacted or retained mandibular second molars, a miniscrew is often fitted in the retromolar region, for tooth traction.

Nanda $^{9}$, for example, described traction of a mandibular second molar impacted horizontally behind the first molar, using a bracket-headed miniscrew in the retromolar region, with a button glued to the distal side of the second molar (the only accessible side at 


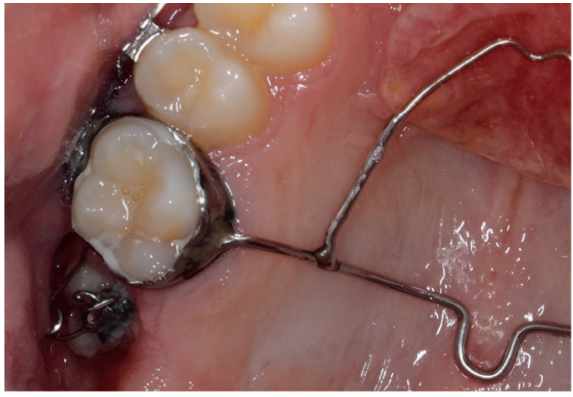

a

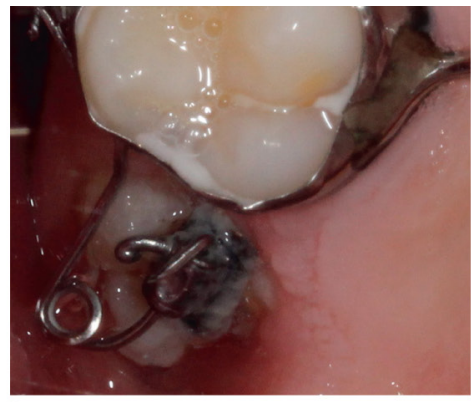

$b$

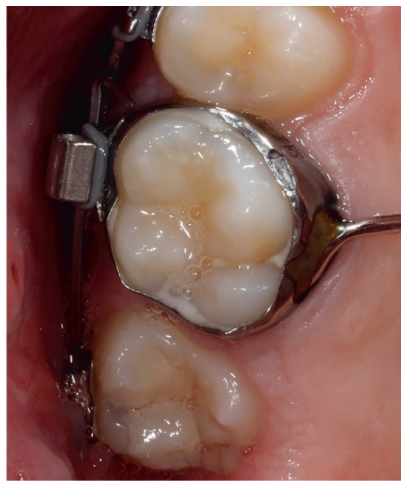

C

Figure 8

a, b) Traction of 17 by a tube glued to the occlusal side and a .017 x.022 sectional bracket with blue elgiloy loops. c) A tube is glued to the vestibular side, with a sectional bracket to the first molar including the tooth under traction. (Thanks to $\operatorname{Dr} A$. Birgy).

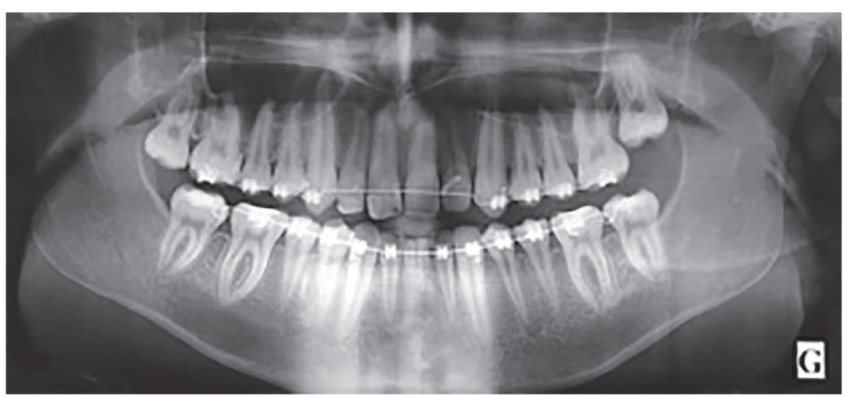

a
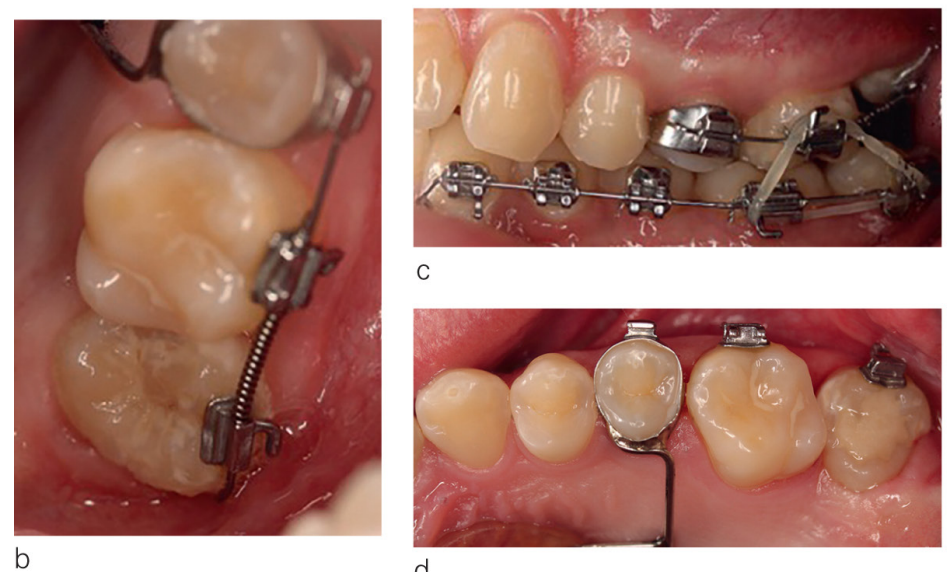

C
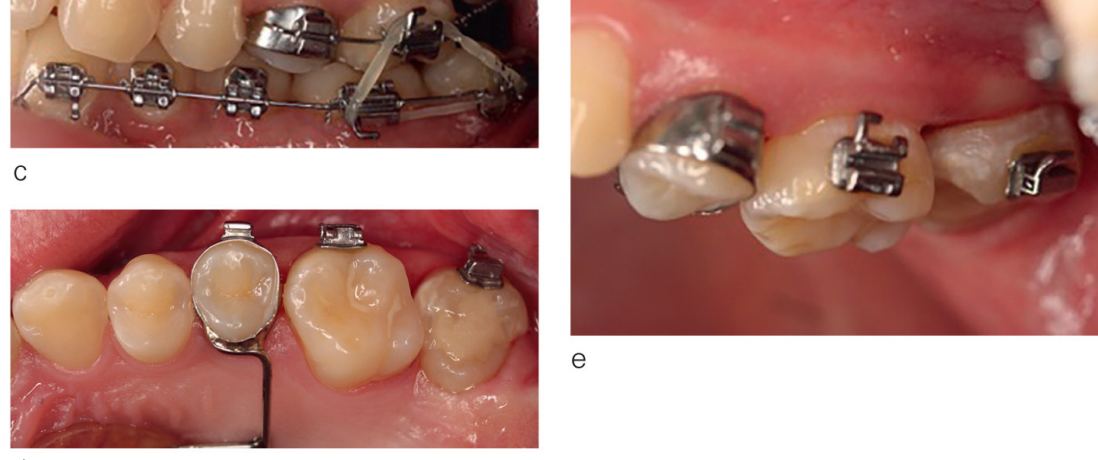

e

d

Figure 9

a) Panoramic view, showing impaction of 27. b) Sectional bracket and spring to distalize 27. c) Triangular elastic to avoid side-effects on adjacent teeth. $d$ and e) Progressive egression of 27: occlusal and lateral views. (Thanks to Dr S. Legris). 


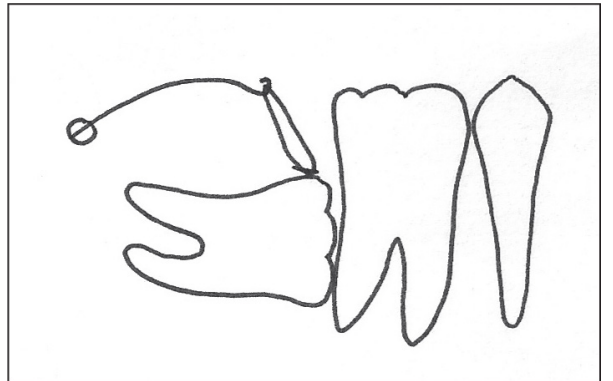

Figure 10

Nanda device.

this stage) and a cantilever mechanism between the miniscrew and an elastic traction band connecting with the second molar button (Fig. 10).

Park et al. ${ }^{11}$ reported using a miniscrew in the maxillary tubercle to upright a maxillary second molar; but in their case the adjacent first molar was absent.

Sohn et al..$^{15}$ stressed the drawbacks of applying distal force on the tooth to be positioned: patient discomfort, and poor efficacy of the system, which can rarely be applied to the maxilla. They recommended an alternative, using a miniscrew between the second premolar and first molar, fixed by a .016 $\times .022$ steel wire and composite. The miniscrew serves as anchorage rather than for traction.

\section{Miniplates and orthodontics}

Tseng et al. ${ }^{17}$ used a miniplate for traction of a severely impacted mandibular second molar. Miniplates are harder to fit than miniscrews, and should be reserved for more complex cases in which miniscrews alone would not allow the impacted tooth to be positioned.

Bone anchorage is of great interest, avoiding parasitic ingression in case of ankylosis.

\section{Exposure and dislocation associated to orthodontics}

A 19 year-old patient presented with a totally edified right maxillary molar, without progression at end of orthodontic treatment; the adjacent third molar was extracted following iterative infection, but this did not enable spontaneous eruption of the second molar (Fig. 11a).

Surgical exposure was associated to dislocation. At the time of writing, the tooth was under traction with a sectional $.016 \times .016$ copper NiTi overlay bracket, with anchorage by a $.018 \times .025$ steel sectional bracket $(.022 \times .028$ attachments $)$ connecting the two premolars and the first molar, plus a transpalatal arch fixed to the two first premolars (Fig. 11b). This type of treatment, associating orthodontic traction to surgical dislocation of the suspected ankylosis region, was described by Dr CohenLévy ${ }^{4}$.

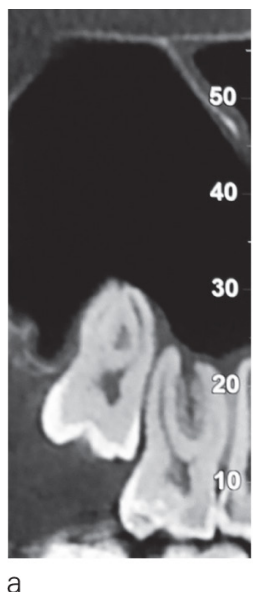

a

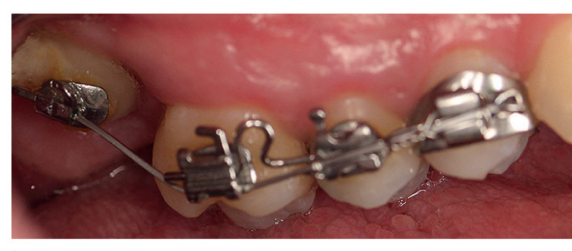

b
Figure 11

a) Cone-beam CT slice of impacted 17. b) Traction of 17 by $.016 \times .016$ copper NiTi sectional bracket. (Thanks to Dr Sylvie Legris). 


\section{Orthodontics after surgical setting}

In some cases, orthodontic treatmentisneededaftersurgerytocorrect, for example, unduly lingual or vestibular positioning ${ }^{5}$; classic multibracket systems, partial or otherwise, may then be used.

\section{CONCLUSION}

The relatively low frequency of second molar eruption disorder explains why treatment is so poorly standardized, without any large-scale studies. The present article shows the diversity of methods: orthodontic and associated orthodontic/surgical, each with its advantages and drawbacks. The importance of good anchorage should be kept in mind, to avoid and anticipate parasitic movements, being fully aware of the effects of the mechanical system used. Close monitoring is essential. Any tooth showing signs of ankylosis can thus be detected before inducing side-effects, which could be non-negligible and difficult to manage subsequently.

\section{Conflicts of interest}

The authors declare no conflicts of interest.

\section{REFERENCES}

1. Bach RM. Non-surgical uprighting of exposed, mesially impacted lower molars. J Clin Orthod 2012;46:480-485.

2. Celli D, Gasperoni E, Pansoni P, Deli R. Uprighting of mandibular second molars with the sectional modified Loca system. Orthodontics(Chic) 2013;14(1):e118-e125.

3. Cho S, Ki Y, Chu V, Chan J. Inclusion des deuxièmes molaires permanentes chez des enfants d'ethnie chinoise d'âge scolaire. JADC 2008;74(6).

4. Cohen-Lévy J. Ankylosis of permanent first molars: Genetics or environment? A case report of a discordant twin pair. Int Orthod 2011;9(1):76-91.

5. Davis WH, Patakas BM, Kaminishi RM, Parsch NE. Surgically uprighting and grafting mandibular second molars. Am J Orthod 1976;69(5):555-561.

6. Lau CK, Whang CZY, Bister D. Orthodontic uprighting of severely impacted mandibular second molars. Am J Orthod Dentofacial Orthop 2013;143(1):116-124.

7. Ma Z, Yang C, Zhang S, Xie Q, Shen Y, Shen P. Orthodontic extrusion of horizontally impacted man- dibular molars. Int J Clin Exp Med 2014;7(10):3320- 3326.

8. Miao YQ, Zhong $\mathrm{H}$. An uprighting appliance for impacted mandibular second and third molars. J Clin Orthod 2006;40(2):110-116.

9. Nanda RS, Tosun YS. Biomechanics in Orthodontics, principles and practice. Hanover Park: Quintessence Publishing Co Inc 2010:141-143.

10. Paik CH, Kim HH, Park YG, Ahn HW. Strategic tooth movement with a light nickel titanium expansion wire. J Clin Orthod 2014;48(5):295-302.

11. Park HS, Kyung HM, Sung JH. A simple method of molar uprighting with microimplant anchorage. J Clin Orthod 2002;36(10):592-596. 
12. Proffit WR, Vig KWL. Primary failure of eruption: a possible cause of posterior open bite. Am J Orthod 1981;80(2):175-190.

13. Santoro M, Kim ES, Teredesai M, Karaggiannopoulos N. Modified removable transpalatal bar for rapid uprighting of impacted second molars. J Clin Orthod 2002;36(9):496-498.

14. Sawicka M, Racka-Pilszak B, Rosnowska-Mazurkiewicz Uprighting partially impacted permanent second molars. Angle Orthod 2007;77(1):148-154.

15. Sohn BW, Choi JH, Jung SN, Lim KS. Uprighting mesially impacted second molars with miniscrew anchorage. J Clin Orthod 2007;41(2):94-97.

16. Suri L, Gagari E, Vastardis H. Delayed tooth eruption: pathogenesis, diagnosis, and treatment. A literature review. Am J Orthod Dentofacial Orthop 2004;126(4):432-445.

17. Tseng $\mathrm{YC}$, Chen $\mathrm{CM}$, Chang HP. Use of a miniplate for skeletal anchorage in the treatment of a severely impacted mandibular second molar. $\mathrm{Br} \mathrm{J}$ Oral Maxillofac Surg 2008;46(5):406-407.

18. Ziegler S. Uprighting mechanics for mesially inclined lower second and third molars. $\mathrm{J}$ Clin Orthod 2012;46(4):218-224. 